\title{
Improving access and equity in reducing cardiovascular risk: the Queensland Health model
}

\section{A ... statewide coaching \\ program ... \\ facilitates a \\ guideline- \\ concordant \\ decrease in \\ cardiovascular \\ risk}

Chantal F Sk

BA(Hons), PhD

Margarite J Vale

PhD, FCSANZ ${ }^{2,3}$

Gary R Bennet

$\mathrm{MN}^{4}$

Victoria L Chalmers

ExecMPublicAdmin GradDipLiblnfoStud, BCom

Kim McFarlane

MBBS, FRACGP

V Michael Jelinek

MBBS, MD, FCSANZ1,

Ian A Scott

FRACP, MHA, MEd

David R Thompson

MA, MBA, PhD

1Centre for the Heart and Mind

Melbourne, VIC.

2 University of Melbourne Melbourne, VIC

3 The COACH Program, Melbourne, VIC

4 Queensland Health. Brisbane, QLD.

5 St Vincent's Hospital, Melbourne, VIC.

6 Princess Alexandra Hospital, Brisbane, QLD.

m.vale@unimelb.edu.au

doi: 10.5694/mjal4.00575
A lmost one-third of all deaths in the Australian population in 2011 were attributable to cardiovascular disease (CVD), ${ }^{1}$ and around $80 \%$ of these were preventable. ${ }^{2}$ People who attend cardiac rehabilitation programs have reduced mortality rates and improved health knowledge, health behaviours and quality of life compared with patients who do not attend rehabilitation. ${ }^{3,4}$ Attendance rates are around $30 \%$ or less and have not improved in the past 20 years ${ }^{5-7}$ despite major attempts by advisory bodies to increase uptake. ${ }^{8,9}$ As a result, alternative methods of delivering cardiac rehabilitation and secondary prevention have been sought. ${ }^{10}$ These include home-based cardiac rehabilitation, case management and, more recently, telephone coaching programs that are flexible, multifaceted and integrated with the patient's primary health care provider. ${ }^{11-13}$ Achieving optimal and sustainable delivery of these interventions to rural and remote communities presents a huge challenge.

Australia's rural population is onethird of the total population and has higher mortality rates, lower life expectancy and higher rehospitalisation rates than its metropolitan counterpart. ${ }^{14}$ Attendance at cardiac rehabilitation is lower in rural and remote communities. ${ }^{15}$ Aboriginal and Torres Strait Islander Australians face an even greater burden of disease because of their increased predisposition for acquiring diabetes (up to 10-fold higher prevalence) ${ }^{16,17}$ and associated cardiovascular risk factors throughout life and at an earlier age than the non-Indigenous population. ${ }^{16,18}$ Targeted outreach programs aimed at increasing equity of access to health services are likely to have the greatest potential to improve population health.

Queensland is Australia's most decentralised state, with more than half of its population living outside

Abstract

Objectives: To measure changes in cardiovascular risk factors among patients with coronary heart disease (CHD) and/or type 2 diabetes enrolled in a centralised statewide coaching program delivered by telephone and mail-out in the public health sector in Queensland.

Design: A population-based audit of cardiovascular risk factor data collected prospectively as part of The $\mathrm{COACH}$ (Coaching Patients On Achieving Cardiovascular Health) Program (TCP) delivered through Queensland Health's Health Contact Centre.

Setting and participants: 1962 patients with CHD and 707 patients with type 2 diabetes who completed TCP from 20 February 2009 to 20 June 2013, of whom 145 were Indigenous Australians.

Main outcome measures: Changes in fasting lipids, fasting glucose, glycosylated haemoglobin levels, blood pressure, body weight, body mass index, smoking, alcohol consumption and physical activity, as measured at entry to and completion of the program.

Results: Statistically significant improvements in cardiovascular risk factor status, from entry to completion of the program, were found across all biomedical and lifestyle factors in patients with CHD and/or type 2 diabetes. For both diseases, improvements in serum lipids, blood glucose, smoking habit and alcohol consumption combined with increases in physical activity were the most notable findings. Similar differences were found in mean change scores in cardiovascular risk factors between Indigenous and non-Indigenous Queenslanders.

Conclusion: A centralised statewide coaching program delivered by telephone and mail-out overcomes obstacles of distance and limited access to health services and facilitates a guideline-concordant decrease in cardiovascular risk.

of the capital city. ${ }^{19}$ Accordingly, Queensland Health is exploring evidence-based, simple and accessible, integrated and sustainable, costeffective health care innovations. ${ }^{20}$

In 2009, Queensland Health introduced The COACH (Coaching Patients On Achieving Cardiovascular Health) Program (TCP) to assist people diagnosed with chronic diseases and to reduce the risk of future complications. The program has been shown to be superior to usual medical care in reducing risk factor levels in two randomised controlled trials. ${ }^{21,22}$

This program is the first standardised coaching program targeting cardiovascular risk factors and delivered by telephone and mail-out to be made available statewide. Every eligible patient in Queensland can have access to the program irrespective of where they live, in accordance with the Queensland Government's strategy for chronic disease. ${ }^{23}$
We report here on changes in the cardiovascular risk factor status of patients enrolled in $\mathrm{TCP}$, including a comparison of changes in risk factors among Indigenous and non-Indigenous enrollees in Queensland.

\section{Methods}

\section{Design and participants}

We analysed cardiovascular risk factor data collected prospectively as part of TCP. The audit data included: blood test results for fasting lipids, fasting glucose in people without diabetes, and glycated haemoglobin $\left(\mathrm{HbA}_{1 c}\right)$ in people with diabetes; two physical measurements - blood pressure and body weight; and self-reported information about three lifestyle factors - smoking, physical activity and alcohol intake. Information about medications (dose, frequency and adherence) was obtained by the coaches. 
Data were collected from two separate cohorts of patients who had a primary diagnosis on admission of either coronary heart disease (CHD) or type 2 diabetes and who were enrolled in the coaching program between 20 February 2009 and 20 June 2013. All enrolled patients provided consent for de-identified data to be used for evaluation and reporting purposes. The audit was approved by the Clinical Governance Committee of the Health Contact Centre, Queensland Health.

\section{The COACH Program}

TCP is a standardised coaching program delivered by telephone and mail-out for people with or at high risk of chronic disease. Trained health professionals ("coaches") coach people to achieve national guidelinerecommended target levels for their particular risk factors and to take the medications as recommended by guidelines for the management of their particular medical condition or conditions. A more detailed description of TCP is given in Appendix 1.

\section{Entry to TCP}

Queensland Health's Health Contact Centre is responsible for the operation of 13 HEALTH, a 24-hour, seven-daya-week statewide service providing access for all Queenslanders to health information, triage and referral. Using 13HEALTH telephone infrastructure, the contact centre also implements 13QUIT to support smoking cessation, the Child Health Line, and TCP to deliver chronic disease management. ${ }^{24}$ Referral to the centre for participation in TCP is accepted from all sources: public hospitals, general practitioners, medical specialists, other health professionals, cardiac rehabilitation services, Quitline and self-referral. Referral can be online or by fax, email, phone or mail. Once patients are referred to the Health Contact Centre, administration assistants initiate contact with patients and book them in for their first coaching session. The patients do not meet the administration assistants or coaches face-to-face at any stage. Patients are coached in a manner identical to that in the clinical trials. $^{21,22}$ TCP is directed to patients who could not or would not attend cardiac rehabilitation.

\section{Training of the coaches}

The Health Contact Centre employs registered nurses as coaches to deliver TCP. Coaches are trained face-to-face for 2 weeks in the principles and practice of TCP and then undergo a formal 12-week preceptorship program. A detailed description of the training of the coaches is in Appendix 1.

\section{TCP software application}

TCP is fully computerised and uses a customised web-based software application for program delivery and evaluation. The software records all relevant patient and risk factor details, generates structured patient letters and contains all key performance indicators including patient uptake, completion rates, achievement of risk factor targets and adherence to recommended medications at entry to and exit from the program.

\section{Statistical analysis}

Descriptive statistics comprised means and standard deviations for continuous variables, and numbers and percentages for categorical variables. The paired samples $t$ test was used to assess the significance of differences in cardiovascular risk factors at entry and completion within each patient group (CHD and type 2 diabetes). The independent samples $t$ test was used to compare mean change in risk factors from entry to completion of TCP between Indigenous and non-Indigenous patients. Bonferroni correction was used to adjust the $P$ value for multiple comparisons. The Pearson $\chi^{2}$ test was used to compare smoking status from entry to completion across patient cohorts. Data were analysed using SPSS version 20 (SPSS Inc).

\section{Results}

During the audit period, 3235 patients enrolled in TCP and 2669 patients (83\%) completed the program, including 145 Indigenous patients. Five hundred and sixty-six patients $(17 \%)$ did not complete the program (26 died during the audit period). Patients had a primary diagnosis of either CHD
1 Comparison of The COACH Program (TCP) cohort with the Queensland population, by remoteness area $^{26}$

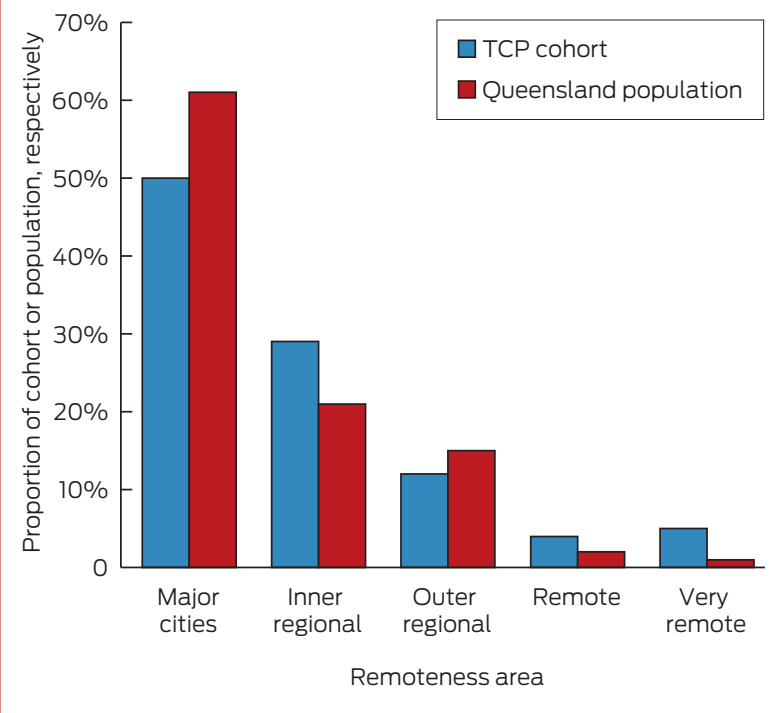

(1962 patients) or type 2 diabetes (707). Fifty-three per cent (1409) had a diagnosis of CVD before the referral event; $59 \%$ of patients with CHD (1158) had a prior history of CVD; and $36 \%$ of patients with type 2 diabetes (251) had CVD as a comorbidity. CVD includes CHD, heart failure, stroke and peripheral vascular disease. Eighty-four per cent of referrals to TCP were made by clinical staff at the time the patient was discharged from a public hospital. Most other referrals were from community health centres or Quitline.

\section{Access and equity}

The Accessibility Remoteness Index of Australia ${ }^{25}$ was used to categorise remoteness (Box 1). The location of this cohort was typical of the location of the general population of Queensland.

\section{Demographics}

Baseline demographics and treatments for patients with CHD or diabetes as their primary diagnosis are listed in Appendix 2. Age within each diagnosis group ranged from 20 to 95 years and from 20 to 90 years, respectively. Both cohorts had the same age:sex ratio as that for the total population of patients with either CHD or diabetes, ${ }^{27}$ and both had the same proportion of Indigenous 
2 Comparison of cardiovascular risk factors from entry to completion of The COACH Program in participants with coronary heart disease $(n=1962)$

Paired differences

\begin{tabular}{|c|c|c|c|c|c|}
\hline \multirow{2}{*}{ Risk factors } & \multirow[b]{2}{*}{ No. of patients* } & \multirow[b]{2}{*}{ Entry, mean (SD) } & \multirow[b]{2}{*}{ Completion, mean (SD) } & \multirow{2}{*}{ Mean $(95 \% \mathrm{Cl})$ of difference } & \multirow[b]{2}{*}{$P$} \\
\hline & & & & & \\
\hline \multicolumn{6}{|l|}{ Fasting lipids, mmol/L } \\
\hline Total cholesterol & 1106 & $4.29(1.27)$ & $3.73(0.96)$ & -0.56 (0.49 to 0.64$)$ & $<0.001$ \\
\hline Triglycerides & 1085 & $1.80(1.84)$ & $1.47(0.97)$ & $-0.33(0.24$ to 0.43$)$ & $<0.001$ \\
\hline HDL cholesterol & 963 & $1.08(0.35)$ & $1.13(0.34)$ & $0.06(-0.07$ to -0.04$)$ & $<0.001$ \\
\hline LDL cholesterol & 951 & $2.44(1.07)$ & $1.83(0.77)$ & -0.61 (0.54 to 0.68$)$ & $<0.001$ \\
\hline Fasting glucose, $\mathrm{mmol} / \mathrm{L}$ & 459 & $5.55(0.90)$ & $5.41(0.92)$ & $-0.14(0.06$ to 0.21$)$ & 0.001 \\
\hline $\mathrm{HbA}_{\mathrm{Ic}}, \%(\mathrm{DM}, n=579)$ & 430 & $7.83 \%(1.80 \%)$ & $7.41 \%(1.43 \%)$ & $-0.43(0.28$ to 0.57$)$ & $<0.001$ \\
\hline BP systolic, mmHg & 1226 & $124.5(16.3)$ & $123.0(13.3)$ & -1.5 (0.67 to 2.42 ) & 0.001 \\
\hline BP diastolic, mmHg & 1221 & $71.4(11.0)$ & $70.2(9.2)$ & -1.2 (0.57 to 1.82 ) & $<0.001$ \\
\hline Body weight, kg & 1864 & $85.1(20.2)$ & $84.1(19.5)$ & -1.1 (0.77 to 1.35$)$ & $<0.001$ \\
\hline $\mathrm{BMI}, \mathrm{kg} / \mathrm{m}^{2}$ & 1632 & $28.8(6.0)$ & $28.5(5.8)$ & -0.3 (0.24 to 0.43$)$ & $<0.001$ \\
\hline Alcohol, standard drinks per day & 1922 & $1.4(1.7)$ & $1.1(1.3)$ & $-0.4(0.29$ to 0.41$)$ & $<0.001$ \\
\hline Physical activity, min/week & 1888 & $142.0(170.3)$ & $229.1(238)$ & $87(-97.30$ to -76.84$)$ & $<0.001$ \\
\hline
\end{tabular}

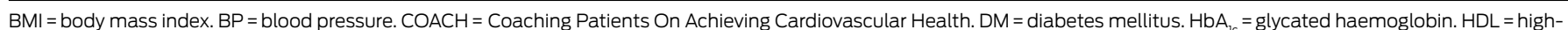

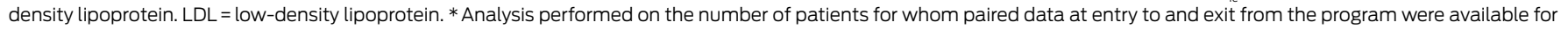
analysis.

Queenslanders as the general population (approximately 6\%). Patients with a primary admission diagnosis of CHD received their first coaching session within 2 months of their index event. Patients received a mean (SD) number of sessions of 5.5 (1.2) over a mean period of 182 days (6 months).

\section{Risk factor changes}

Data for individual risk factors were compared at entry to and completion of TCP in patients for whom complete data were available (for smoking, alcohol intake, physical activity: $96 \%-99 \%$ of patients; for body weight: $83 \%$; for lipid levels, blood pressure, $\mathrm{HbA}_{1 \mathrm{c}}$ levels in patients with diabetes: $48 \%-74 \%$ ). For risk factors where less than $70 \%$ of patients had data at entry and completion, the patients with paired data were compared with the patients without paired data for age, sex and comorbidities and no significant differences were found between the groups. This supports the assumption that missing data occurred at random with no patient selection bias in the pairing of measurements.

\section{Patients with CHD}

Box 2 lists the mean differences between entry and completion for cardiovascular risk factors in patients with CHD. All changes in risk factors remained statistically significant after multiple comparison correction. The direction of mean change in risk factor results showed improvement across all risk factors. The most clinically significant changes were a decrease in mean low-density lipoprotein (LDL) cholesterol level from $2.4 \mathrm{mmol} / \mathrm{L}$ to $1.8 \mathrm{mmol} / \mathrm{L}$, a decrease in mean $\mathrm{HbA}_{1 \mathrm{c}}$ level from $7.8 \%$ to $7.4 \%$, a decrease in mean alcohol intake from 1.4 to 1.1 standard drinks per day, and an increase in mean physical activity from 142 minutes to 229 minutes per week $(P<0.001$ for all comparisons).

\section{Patients with type 2 diabetes}

Similarly, Box 3 lists the results for changes in risk factors in patients with type 2 diabetes, also showing statistically significant improvements in all risk factors after adjustment for multiple comparisons. Again, the most clinically significant changes were a decrease in mean LDL cholesterol (from $2.5 \mathrm{mmol} / \mathrm{L}$ to $2.0 \mathrm{mmol} / \mathrm{L}$ ), a decrease in mean $\mathrm{HbA}_{1 \mathrm{c}}$ (from $8.2 \%$ to $7.5 \%$ ), a decrease in mean alcohol intake (from 1.3 to 0.9 standard drinks per day), and an increase in physical activity (from 127 to 182 minutes per week) $(P<0.001$ for all comparisons).

\section{Smokers}

There were significant decreases in the numbers of current smokers from entry to completion of TCP among patients with CHD (from 296 to 222 $\left.\left[\chi_{(1, n=296)}^{2}=755 ; P<0.001\right]\right)$ and among patients with diabetes (from 139 to $105\left[\chi_{(1, n=139)}^{2}=467 ; P<0.001\right]$.

\section{Indigenous patients}

At entry to TCP, Indigenous patients were younger, consumed more alcohol and were more likely to smoke than non-Indigenous patients; and Indigenous patients with CHD had a lower frequency of percutaneous coronary intervention than non-Indigenous patients (Appendix 3).

There were no significant differences between these two populations for changes in any of the risk factors from entry to completion of TCP.

\section{Medication changes}

Patients' use of statins, $\beta$-blockers, antiplatelet agents and angiotensinconverting enzyme inhibitors or angiotensin receptor antagonists is detailed in Appendix 4. For each agent, there was a highly significant improvement in use between 
3 Comparison of cardiovascular risk factors from entry to completion of The COACH Program in participants with type 2 diabetes $(n=707)$

Paired differences

\begin{tabular}{|c|c|c|c|c|c|}
\hline \multirow[b]{2}{*}{ Risk factors } & \multirow[b]{2}{*}{ No. of patients* } & \multirow[b]{2}{*}{ Entry, mean (SD) } & \multirow[b]{2}{*}{ Completion, mean (SD) } & \\
\hline & & & & Mean $(95 \% \mathrm{Cl})$ of difference & $P$ \\
\hline \multicolumn{6}{|l|}{ Fasting lipids, mmol/L } \\
\hline Total cholesterol & 492 & $4.51(1.26)$ & $4.04(1.02)$ & $-0.47(0.36$ to 0.58$)$ & $<0.001$ \\
\hline Triglycerides & 409 & $2.23(1.84)$ & $1.90(1.24)$ & -0.33 (0.19 to 0.47$)$ & $<0.001$ \\
\hline HDL cholesterol & 404 & $1.06(0.30)$ & $1.09(0.30)$ & $0.04(-0.06$ to -0.01$)$ & 0.001 \\
\hline LDL cholesterol & 396 & $2.46(1.00)$ & $2.04(0.84)$ & $-0.42(0.33$ to 0.51$)$ & $<0.001$ \\
\hline $\mathrm{HbA}_{1 \mathrm{c}}, \%$ & 564 & $8.15 \%(2.06 \%)$ & $7.45 \%(1.54 \%)$ & -0.70 (0.55 to 0.86$)$ & $<0.001$ \\
\hline BP systolic, mmHg & 394 & $129.3(15.7)$ & $127.3(12.8)$ & $-2.0(0.42$ to 3.53$)$ & $<0.001$ \\
\hline BP diastolic, mmHg & 394 & $74.8(9.9)$ & $73.4(9.5)$ & $-1.4(0.39$ to 2.44$)$ & $<0.001$ \\
\hline Body weight, kg & 666 & $101.7(28.5)$ & $100.7(28.1)$ & -1.0 (0.56 to 1.47$)$ & $<0.001$ \\
\hline $\mathrm{BMI}, \mathrm{kg} / \mathrm{m}^{2}$ & 555 & $35.1(9.5)$ & $34.7(9.1)$ & $-0.4(0.18$ to 0.64$)$ & $<0.001$ \\
\hline Alcohol, standard drinks per day & 693 & $1.3(2.0)$ & $0.9(1.5)$ & $-0.4(0.30$ to 0.50$)$ & $<0.001$ \\
\hline Physical activity, min/week & 684 & $127.0(197)$ & $181.6(177.1)$ & $54.7(-68.14$ to -41.17$)$ & $<0.001$ \\
\hline
\end{tabular}

$\mathrm{BMl}=$ body mass index. $\mathrm{BP}=$ blood pressure. $\mathrm{COACH}=$ Coaching Patients On Achieving Cardiovascular Health. $\mathrm{HbA} \mathrm{A}_{1 \mathrm{c}}=$ glycated haemoglobin. $\mathrm{HDL}=$ high-density lipoprotein. $\mathrm{LDL}=$ low-density lipoprotein. *Analysis performed on number of patients for whom paired data at entry to and exit from the program were available for analysis.

entry and completion of TCP for Indigenous and non-Indigenous patients. The most notable improvement was the increase in statin use among patients with diabetes, from $66 \%$ to $78 \%$ overall and from $83 \%$ to $92 \%$ among Indigenous people $(P<0.001$ for both comparisons).

Comparison of results for Indigenous and non-Indigenous patients showed no significant differences for changes in medication use.

\section{Discussion}

People with CHD and/or type 2 diabetes have a markedly higher risk of future cardiac events than the general population and therefore constitute the most appropriate target group for risk-mitigation strategies. Participation in TCP resulted in improvements across all cardiovascular risk factors from entry to completion for patients with CHD and/or type 2 diabetes. Improvements in lipid levels, glucose levels, smoking habit and alcohol consumption combined with increases in physical activity were the most notable findings. Similar results were reported in two previous trials of TCP that assessed cardiovascular risk factor status in patients with CHD. ${ }^{21,22}$ A real-world experience of TCP in 5544 patients with CHD achieved comparable improvement in risk factors. ${ }^{28}$

\section{Study strengths and limitations}

The audit we report involved a statewide population of patients enrolled into TCP over 4 years. Its strengths include the statewide cohort, comparison of Indigenous and nonIndigenous people, measurement of multiple risk factors and data on medication use.

We note limitations with regard to missing patient data for variables that relied on patients' usual doctors measuring the requisite information at entry to and exit from the program. More data were missing at entry because patients may not have visited their doctor to have their risk factors measured before the first coaching session. Results were also missing at exit if requests for repeat pathology tests (such as lipid, glucose and $\mathrm{HbA}_{1 \mathrm{c}}$ levels) were made within a 6-month period, contrary to Medicare guidelines. Again, patients may not have attended their doctor to have their risk factors measured. Among participants for whom paired data were available, all risk factors improved significantly from entry to completion of TCP. Post-hoc analysis confirmed that the missing data were randomly distributed in both pre- and post-TCP datasets. Thus, no patient characteristics were identified that would have introduced selection bias. A final limitation was the reliance on unverified patient self-report for lifestyle measures of smoking, alcohol intake and physical activity.

\section{Potential of The $\mathrm{COACH}$ Program}

Overall, our results provide further evidence to support this intervention as an effective strategy for reducing cardiovascular risk and for secondary prevention. As our cohort comprised patients with CHD and/or type 2 diabetes, the results suggest the potential for TCP to be adapted for other chronic diseases.

Exclusive use of telephone and mailouts is unique to the chronic disease management model adopted by Queensland Health. These methods eliminate barriers often seen with cardiac rehabilitation programs, 5,6,10 including geographic isolation, travel costs and the inconvenience of appointments. More sessions could be given by telephone coaching than could have been given face-to-face.

This study is the first to compare effects of a cardiovascular risk factor reduction program between Indigenous and non-Indigenous populations. Even though there were significant differences in baseline demographics and risk factors, 
we found no significant differences between the two populations in the changes in any of the risk factors or in medication adherence after TCP. Similar reductions in risk factors in both groups (regardless of risk profile on entry) suggests TCP can be successfully applied across different populations and cultures.

Currently, the components common to most cardiac rehabilitation programs across Australia comprise exercise and education, although exactly how these are delivered in terms of frequency and intensity is not well defined and varies between programs, with no consistent measurement of changes in cardiovascular risk factors. TCP is delivered by trained coaches using a standardised model of care based on risk assessments and goal setting that reflects national disease management guidelines. TCP could be extended nationwide and its effects on risk factor profiles, clinical events, physical function and quality of life could be assessed using national surveys and routinely collected data.

\section{Conclusion}

TCP delivered by telephone and mail-outs offers equitable access to an effective health service to all Queenslanders with CHD and/or diabetes, irrespective of their geographic location and ability to access formal cardiac rehabilitation or diabetes education programs. The methods of TCP validated in previous randomised controlled trials ${ }^{21,22}$ have been applied to routine practice and made available to a widely dispersed population, including residents of remote areas and Indigenous peoples. Given the burden of CHD and diabetes, it offers a sustainable means for optimising health outcomes across diverse populations.

Competing interests: Gary Bennett is the Nurse Unit Manager of the Chronic Disease Service that delivers The COACH Program in Queensland Health. Victoria Chalmers has management responsibilities and accountabilities for The COACH Program service delivery within the Health Contact Centre, Department of Health. Margarite Vale is the director of The COACH Program. The data used in this study are owned by Queensland Health. The data were collected by Queensland Health as part of the usual care of delivering The COACH Program.

Received 15 Apr 2014, accepted 9 Sep 2014. 
1 Australian Bureau of Statistics. Causes of death, Australia, 2011. Canberra: ABS, 2013. (ABS Cat. No. 3303.0.) http:// www.abs.gov.au/ausstats/abs@.nsf/ Lookup/3303.0Chapter42011 (accessed Sep 2014).

2 World Health Organization. Cardiovascular diseases. Data and statistics. http://www.euro.who.int/ en/health-topics/noncommunicablediseases/cardiovascular-diseases/ data-and-statistics (accessed Feb 2014).

3 Heran BS, Chen JM, Ebrahim S, et al. Exercise-based cardiac rehabilitation for coronary heart disease. Cochrane Database Syst Rev 2011; (7): CD001800.

4 Leon AS, Franklin BA, Costa F, et al. Cardiac rehabilitation and secondary prevention of coronary heart disease: an American Heart Association scientific statement from the Council on Clinical Cardiology (Subcommittee on Exercise, Cardiac Rehabilitation, and Prevention) and the Council on Nutrition, Physical Activity, and Metabolism (Subcommittee on Physical Activity), in collaboration with the American association of Cardiovascular and Pulmonary Rehabilitation. Circulation 2005; 111 : 369-376.

5 Bunker SJ, Goble AJ. Cardiac rehabilitation: under-referral and underutilisation. Med J Aust 2003; 179: 332-333.

6 Thompson DR, Clark AM. Cardiac rehabilitation: into the future. Heart 2009; 95: 1897-1900.

7 Scott IA, Lindsay KA, Harden HE. Utilisation of outpatient cardiac rehabilitation in Queensland. Med J Aust 2003; 179: 341-345.

8 Australian Cardiac Rehabilitation Association. A practitioner's guide to cardiac rehabilitation. Melbourne: ACRA, 1999.

9 National Heart Foundation of Australia, Australian Cardiac Rehabilitation Association. Recommended framework for cardiac rehabilitation '04. 2004. http://www.heartfoundation.org. au/SiteCollectionDocuments/ Recommended-framework.pdf (accessed Sep 2014).
10 Briffa TG, Tonkin A. Put disease prevention first. Circulation 2013; 128 : 573-575.

11 Redfern J, Chow CK. Secondary prevention of coronary heart disease in Australia: a blueprint for reform. Med J Aust 2013; 198: 70-71.

12 Briffa TG, Kinsman L, Maiorana AJ, et al. An integrated and coordinated approach to preventing recurrent coronary heart disease events in Australia. Med J Aust 2009; 190: 683-686.

13 Riley DL, Stewart DE, Grace SL. Continuity of cardiac care: cardiac rehabilitation participation and other correlates. Int J Cardiol 2007; 119: 326-333.

14 Australian Institute of Health and Welfare. Cardiovascular medicines and primary health care: a regional analysis. Canberra: AlHW, 2010. (AlHW Cat. No. CVD 48; Cardiovascular Disease Series No. 32.) http://www.aihw.gov.au/ publication-detail/?id=6442468337 (accessed Sep 2014).

15 De Angelis C, Bunker S, Schoo A. Exploring the barriers and enablers to attendance at rural cardiac rehabilitation programs. Aust J Rural Health 2008; 16: 137-142.

16 Burke V, Zhao Y, Lee AH, et al. Predictors of type 2 diabetes and diabetes-related hospitalisation in an Australian Aboriginal cohort. Diabetes Res Clin Pract 2007; 78: 360-368.

17 McDermott R, Rowley KG, Lee AJ, et al. Increase in prevalence of obesity and diabetes and decrease in plasma cholesterol in a central Australian Aboriginal community. Med J Aust 2000; 172: 480-484.

18 Leonard D, McDermott R, Odea K, et al. Obesity, diabetes and associated cardiovascular risk factors among Torres Strait Islander people. Aust NZ J Public Health 2002; 26: 144-149.

19 Queensland Health. Safe applicable healthcare for rural and remote Queensland - an implementation plan. http://www.health.qld.gov.au/ caru/networks/docs/srrcn-samohimplementation.pdf (accessed Aug 2014).

20 Queensland Health. Blueprint for better healthcare in Queensland. Brisbane:
Queensland Health, 2013. https:// publications.qld.gov.au/storage/ f/2014-06-03T23\%3A46\%3A13.459Z/ blueprint-for-better-healthcare-screen. pdf (accessed Aug 2014).

21 Vale MJ, Jelinek MV, Best JD, Santamaria JD. Coaching patients with coronary heart disease to achieve the target cholesterol: a method to bridge the gap between evidencebased medicine and the "real world" - randomized controlled trial. J Clin Epidemiol 2002; 55: 245-252.

22 Vale MJ, Jelinek MV, Best JD, et al. Coaching patients On Achieving Cardiovascular Health (COACH): a multicenter randomized trial in patients with coronary heart disease. Arch Intern Med 2003; 163: 2775-2783.

23 Queensland Health. Queensland strategy for chronic disease 20052015: framework for self-management 2008-2015. Brisbane: Queensland Health, 2008.

24 Health Support Queensland. Business profile. Health Contact Centre. March 2014. www.health.qld.gov.au/hsq/ about/hcc-business-profile.pdf (accessed Sep 2014).

25 Queensland Government, Queensland Treasury and Trade. Accessibility/ Remoteness Index of Australia. 2013. http://www.oesr.qld.gov.au/aboutstatistics/statistical-standards/ national/aria.php (accessed Dec 2013).

26 Australian Bureau of Statistics. Regional population growth, Australia, 2011. Canberra: ABS, 2012. (ABS Cat. No. 3218.0.) http://www.abs.gov.au/ ausstats/abs@.nsf/Products/3218.0 2 011 Main+Features Main+Features?0 penDocument\#PARALINK3 (accessed Jul 2014).

27 Jousilahti V, Tuomilehto P. Sex, age, cardiovascular risk factors, and coronary heart disease: a prospective follow-up study of 14786 middle-aged men and women in Finland. Circulation 1999; 99: 1165-1172.

28 Jelinek MV, Santamaria JD, Thompson DR, Vale MJ. 'Fit for purpose'. The COACH Program improves lifestyle and biomedical cardiac risk factors. Heart 2012; 98: 1608. 